Pacific

Journal of

Mathematics

\title{
SUPERLINEAR PROBLEMS
}

Martin Schechter and Wenming Zou 


\title{
SUPERLINEAR PROBLEMS
}

\section{Martin Schechter AND Wenming Zou}

\begin{abstract}
We solve elliptic semilinear boundary value problems in which the nonlinear term is superlinear. By weakening the hypotheses, we are able to include more equations than hitherto permitted. In particular, we do not require the superquadracity condition imposed by most authors, and it is not assumed that the region is bounded.
\end{abstract}

\section{Introduction.}

Consider the problem

$$
-\Delta u=f(x, u), x \in \Omega ; \quad u=0 \text { on } \partial \Omega,
$$

where $\Omega \subset \mathbb{R}^{n}$ is a bounded domain whose boundary is a smooth manifold, and $f(x, t)$ is a continuous function on $\bar{\Omega} \times \mathbb{R}$. This semilinear Dirichlet problem has been studied by many authors. It is called sublinear if there is a constant $C$ such that

$$
|f(x, t)| \leq C(|t|+1), \quad x \in \Omega, t \in \mathbb{R} .
$$

Otherwise, it is called superlinear. Beginning in [1], almost all researchers studying the superlinear problem assumed:

$\left(\mathrm{a}_{1}\right)$ There are constants $c_{1}, c_{2} \geq 0$ such that

$$
|f(x, t)| \leq c_{1}+c_{2}|t|^{s}
$$

where $0 \leq s<(n+2) /(n-2)$ if $n>2$.

$\left(\mathrm{a}_{2}\right) f(x, t)=o(|t|)$ as $t \rightarrow 0$.

(a3) There are constants $\mu>2, r \geq 0$ such that

$$
0<\mu F(x, t) \leq t f(x, t), \quad|t| \geq r,
$$

where

$$
F(x, t)=\int_{0}^{t} f(x, s) d s
$$

They proved:

Theorem 1.1. Under Hypotheses $\left(\mathrm{a}_{1}\right)-\left(\mathrm{a}_{3}\right)$, Problem (1) has a nontrivial weak solution. 
Condition $\left(\mathrm{a}_{3}\right)$ is convenient, but it is very restrictive. In particular, it implies that there exist positive constants $c_{3}, c_{4}$ such that

$$
F(x, t) \geq c_{3}|t|^{\mu}-c_{4}, \quad x \in \Omega, t \in \mathbb{R} .
$$

Although this condition is weaker, it still eliminates many superlinear problems.

A much weaker condition that implies superlinearity is

$\left(a_{3}^{\prime}\right)$ Either

$$
F(x, t) / t^{2} \rightarrow \infty \text { as } t \rightarrow \infty
$$

or

$$
F(x, t) / t^{2} \rightarrow \infty \text { as } t \rightarrow-\infty
$$

The purpose of the present paper is to explore what happens when $\left(a_{3}\right)$ is replaced with $\left(a_{3}^{\prime}\right)$. Surprisingly, we find the following to be true:

Theorem 1.2. Under Hypotheses $\left(\mathrm{a}_{1}\right),\left(\mathrm{a}_{2}\right)$ and $\left(\mathrm{a}_{3}^{\prime}\right)$ the boundary value problem

$$
-\Delta u=\beta f(x, u), x \in \Omega ; \quad u=0 \text { on } \partial \Omega,
$$

has a nontrivial solution for almost every positive $\beta$.

Unfortunately, this theorem does not give any information for any specific $\beta$. We then turned our attention to solving (1) under a weaker assumption than $\left(\mathrm{a}_{3}\right)$. For this purpose we introduced:

$\left(\mathrm{a}_{3}^{\prime \prime}\right)$ There are constants $\mu>2, r \geq 0$ such that

$$
\mu F(x, t)-t f(x, t) \leq C\left(t^{2}+1\right), \quad|t| \geq r .
$$

Note that $\left(\mathrm{a}_{3}\right)$ implies both $\left(\mathrm{a}_{3}^{\prime}\right)$ and $\left(\mathrm{a}_{3}^{\prime \prime}\right)$, but they are much weaker. We prove:

Theorem 1.3. Under Hypotheses $\left(\mathrm{a}_{1}\right),\left(\mathrm{a}_{2}\right),\left(\mathrm{a}_{3}^{\prime}\right)$ and $\left(\mathrm{a}_{3}^{\prime \prime}\right)$ Problem (1) has a nontrivial solution.

It should be noted that the first option in $\left(a_{3}^{\prime}\right)$ together with (5) implies $(3)$.

We also have:

Theorem 1.4. If we replace Hypothesis $\left(\mathrm{a}_{3}^{\prime \prime}\right)$ with:

$\left(\mathrm{a}_{3}^{\prime \prime \prime}\right)$ The function

$$
H(x, t):=t f(x, t)-2 F(x, t)
$$

is convex in $t$,

then Problem (1) has at least one nontrivial solution. 
Costa-Magalhães [2] solved (1) under the following assumptions:

$$
\begin{gathered}
|f(x, t)| \leq a_{0}|t|^{p-1}+b_{0}, \quad x \in \Omega, t \in \mathbb{R}, \\
\limsup _{|t| \rightarrow \infty} \frac{F(x, t)}{|t|^{q}} \leq b<\infty \quad \text { uniformly for a.e. } x \in \Omega, \\
\liminf _{|t| \rightarrow \infty} \frac{H(x, t)}{|t|^{\mu}} \geq a>0 \quad \text { uniformly for a.e. } x \in \Omega, \\
\limsup _{t \rightarrow 0} 2 F(x, t) / t^{2} \leq \alpha<\lambda_{0} \quad \text { uniformly for a.e. } x \in \Omega, \\
F(x, t) / t^{2} \rightarrow \infty \text { as }|t| \rightarrow \infty,
\end{gathered}
$$

where $1 \leq p<2 n /(n-2), \mu>n(q-2) / 2$.

Willem-Zou [11] proved a weaker form of Theorem 1.2 for a special case. They do not require Hypothesis ( $\left.\mathrm{a}_{3}\right)$, but they do assume

$$
t f(x, t) \geq 0, \quad t \in \mathbb{R}
$$

and

$$
t f(x, t) \geq c_{0}|t|^{\mu}, \quad|t| \geq r
$$

for some constants $c_{0}>0, r \geq 0, \mu>2$. Some authors have replaced Hypothesis $\left(\mathrm{a}_{3}\right)$ with (3). Although (3) is a more natural assumption, it is still too restrictive to be desirable. It is for this reason that we introduced assumptions $\left(a_{3}^{\prime}\right)$ and $\left(a_{3}^{\prime \prime}\right)$.

Stronger versions of Theorems 1.2-1.4 will be given in the next section. In them we are not restricted to any particular boundary value problem, and it is not assumed that the region $\Omega$ is bounded.

\section{The main theorems.}

Many elliptic semilinear problems can be described in the following way: Let $\Omega$ be a domain in $\mathbf{R}^{n}$, and let $A$ be a selfadjoint operator on $L^{2}(\Omega)$. We assume that $A \geq \lambda_{0}>0$ and that

$$
C_{0}^{\infty}(\Omega) \subset D:=D\left(A^{1 / 2}\right) \subset H^{m, 2}(\Omega)
$$

for some $m>0$, where $C_{0}^{\infty}(\Omega)$ denotes the set of test functions in $\Omega$ (i.e., infinitely differentiable functions with compact supports in $\Omega$ ) and $H^{m, 2}(\Omega)$ denotes the Sobolev space. If $m$ is an integer, the norm in $H^{m, 2}(\Omega)$ is given by

$$
\|u\|_{m, 2}:=\left(\sum_{|\mu| \leq m}\left\|D^{\mu} u\right\|^{2}\right)^{1 / 2} .
$$

Here $D^{\mu}$ represents the generic derivative of order $|\mu|$ and the norm on the right-hand side of $(8)$ is that of $L^{2}(\Omega)$. If $m$ is not an integer, there are 
several ways of defining the space $H^{m, 2}(\Omega)$, all of which are equivalent. We shall not assume that $m$ is an integer.

A typical example of an operator $A$ satisfying these hypotheses is a second order elliptic operator with smooth coefficients applied to functions satisfying zero Dirichlet boundary conditions on a smooth bounded domain in $\mathbb{R}^{n}$. Only the abstract properties listed above are relevant to our analysis.

Let $q$ be a number satisfying

$$
\begin{array}{ll}
2<q \leq 2 n /(n-2 m), & 2 m<n, \\
2<q<\infty, & n \leq 2 m,
\end{array}
$$

and let $f(x, t)$ be a Carathéodory function on $\Omega \times \mathbf{R}$. This means that $f(x, t)$ is continuous in $t$ for a.e. $x \in \Omega$ and measurable in $x$ for every $t \in \mathbf{R}$.

We consider the problem

$$
A u=f(x, u), u \in D .
$$

By a solution of (10) we shall mean a function $u \in D$ such that

$$
(u, v)_{D}=(f(\cdot, u), v), \quad v \in D .
$$

If $u$ is a solution of $(11)$ and $f(x, u)$ is in $L^{2}(\Omega)$, then $u$ is in $D(A)$ and solves (10) in the classical sense. Otherwise we call it a weak (or semistrong) solution.

We make the following assumptions:

(A) The function $f(x, t)$ satisfies

$$
|f(x, t)| \leq V(x)^{q}\left(|t|^{q-1}+1\right)
$$

and

where $V(x)>0$ is a function in $L^{q}(\Omega)$ such that

$$
f(x, t) / V(x)^{q}=o\left(|t|^{q-1}\right) \text { as }|t| \rightarrow \infty,
$$

(1)

$$
\|V u\|_{q} \leq C\|u\|_{D}, \quad u \in D
$$

Here

$$
\begin{gathered}
\|u\|_{q}:=\left(\int_{\Omega}|u(x)|^{q} d x\right)^{1 / q}, \\
\|u\|_{D}:=\left\|A^{1 / 2} u\right\|,
\end{gathered}
$$

and $q^{\prime}=q /(q-1)$. If $\Omega$ and $V(x)$ are bounded, then (14) will hold automatically by the Sobolev inequality. However, there are functions $V(x)$ which are unbounded and such that (14) holds even on 
unbounded regions $\Omega$ (cf., e.g., [4]). With the norm (16), $D$ becomes a Hilbert space.

(B) The point $\lambda_{0}$ is an isolated simple eigenvalue with a bounded eigenfunction $\varphi_{0}(x) \neq 0$ a.e. in $\Omega$.

(C) There is a $\delta>0$ such that

$$
2 F(x, t) \leq \lambda_{0} t^{2}, \quad|t| \leq \delta, \quad x \in \Omega,
$$

where

$$
F(x, t):=\int_{0}^{t} f(x, s) d s .
$$

(D) There is a function $W(x) \in L^{1}(\Omega)$ such that either

$$
W(x) \leq F(x, t) / t^{2} \rightarrow \infty \text { as } t \rightarrow \infty, \quad x \in \Omega
$$

or

$$
W(x) \leq F(x, t) / t^{2} \rightarrow \infty \text { as } t \rightarrow-\infty, \quad x \in \Omega .
$$

(The function $W(x)$ need not be positive.)

(E) There are constants $\mu>2, C \geq 0$ such that

$$
[\mu F(x, t)-t f(x, t)] /\left(t^{2}+1\right) \leq C, \quad t \in \mathbb{R}, \quad x \in \Omega .
$$

We shall prove:

Theorem 2.1. Under the above hypotheses, the problem

$$
A u=f(x, u), u \in D
$$

has at least one nontrivial solution.

We also have:

Theorem 2.2. Replace Hypothesis (E) with:

$\left(\mathrm{E}^{\prime}\right)$ The function

$$
H(x, t):=t f(x, t)-2 F(x, t)
$$

is convex in $t$.

Then Problem (18) has at least one nontrivial solution.

Problem (18) is called sublinear if $f(x, t)$ satisfies

$$
|f(x, t)| \leq C(|t|+1), \quad x \in \Omega, t \in \mathbb{R} .
$$

Otherwise it is called superlinear. Hypothesis (D) requires (18) to be superlinear.

Problem (18) has been studied by many people. The vast majority of results obtained concern sublinear problems. Much less has been proved for the superlinear case. In [1] the basic assumption was

$$
0<\mu F(x, t) \leq t f(x, t), \quad|t| \geq r
$$


for some $\mu>2$ and $r \geq 0$. This is a very convenient hypothesis since it readily achieves mountain pass geometry as well as satisfaction of the Palais-Smale condition. However it is a severe restriction; it strictly controls the growth of $f(x, t)$ as $|t| \rightarrow \infty$. Almost every author discussing superlinear problems has made this assumption. We have been able to weaken this assumption considerably, but not to our complete satisfaction. We assume either that

$$
\mu F(x, t)-t f(x, t) \leq C\left(t^{2}+1\right), \quad|t| \geq r
$$

for some $\mu>2$ and $r \geq 0$ or that (19) is convex in $t$. These allow much more freedom for the function $f(x, t)$. But they do not allow as much freedom as we would like.

If we drop Hypothesis (E) completely, then we are able to prove the following theorems:

Theorem 2.3. If we replace Hypotheses (C) and (D) with:

$\left(\mathrm{C}^{\prime}\right)$ There are $a \delta>0$ and $a \tilde{\lambda}>\lambda_{0}$ such that

$$
2 F(x, t) \geq \widetilde{\lambda} t^{2}, \quad|t| \leq \delta, x \in \Omega,
$$

and

$\left(\mathrm{D}^{\prime}\right)$ there is a function $W(x) \in L^{1}(\Omega)$ such that

$$
W(x) \geq P(x, t) \rightarrow-\infty \text { as }|t| \rightarrow \infty, \quad x \in \Omega,
$$

where

$$
P(x, t):=F(x, t)-\frac{1}{2} \lambda_{0} t^{2},
$$

and drop Hypothesis (E), then Problem (18) has at least one nontrivial solution.

We also have:

Theorem 2.4. Assume that (A)-(D) hold. Then for almost every $\beta \in$ $(0,1)$, the equation

$$
A u=\beta f(x, u)
$$

has a nontrivial solution. In particular, the eigenvalue problem (23) has infinitely many solutions.

Theorem 2.5. Replace Hypothesis (C) in Theorem 2.4 with:

$\left(\mathrm{C}^{\prime \prime}\right)$ There are $\delta>0$ and $\widetilde{\lambda} \leq \lambda_{0}$ such that

$$
2 F(x, t) \leq \widetilde{\lambda} t^{2}, \quad|t| \leq \delta, x \in \Omega,
$$

and (D) with: 
$\left(D^{\prime \prime}\right)$ Either

$$
\int_{\Omega} F\left(x, R \varphi_{0}\right) d x / R^{2} \rightarrow \infty \text { as } R \rightarrow \infty
$$

or

$$
\int_{\Omega} F\left(x,-R \varphi_{0}\right) d x / R^{2} \rightarrow \infty \text { as } R \rightarrow \infty .
$$

Then (23) has a nontrivial solution for almost every $\beta \in\left(0, \lambda_{0} / \widetilde{\lambda}\right)$.

Corollary 2.6. Replace Hypothesis $\left(\mathrm{C}^{\prime \prime}\right)$ in Theorem 2.5 with:

$\left(\mathrm{C}^{\prime \prime \prime}\right) F(x, t) / t^{2} \rightarrow 0$ uniformly as $t \rightarrow 0$.

Then (23) has a nontrivial solution for almost every $\beta \in(0, \infty)$.

The method (called the monotonicity trick) which allows one to solve (23) for almost all values of $\beta$ in some interval was first introduced by Struwe [8] for minimization problems. It was applied by Jeanjean [3] and others for various types of problems.

\section{Preliminaries.}

Define

$$
G(u):=\|u\|_{D}^{2}-2 \int_{\Omega} F(x, u) d x .
$$

Under Hypothesis (A), it is known that $G$ is a continuously differentiable functional on the whole of $D$. In fact, the following were proved in $[\mathbf{5}$, pp. $56-58]$ :

Proposition 3.1. Under Hypothesis (A), $F(x, u(x))$ and $v(x) f(x, u(x))$ are in $L^{1}(\Omega)$ whenever $u, v \in D$.

Proposition 3.2. $G(u)$ has a Fréchet derivative $G^{\prime}(u)$ on $D$ given by

$$
\left(G^{\prime}(u), v\right)_{D}=2(u, v)_{D}-2(f(\cdot, u), v) .
$$

Proposition 3.3. The derivative $G^{\prime}(u)$ given by $(25)$ is continuous in $u$.

Theorem 3.4. Under Hypotheses (A)-(C), the following alternative holds: Either:

(a) There is an infinite number of $y(x) \in D(A) \backslash\{0\}$ such that

$$
A y=f(x, y)=\lambda_{0} y,
$$

or

(b) for each $\rho>0$ sufficiently small, there is an $\varepsilon>0$ such that

$$
G(u) \geq \varepsilon, \quad\|u\|_{D}=\rho .
$$




\section{Proofs.}

Proof of Theorem 2.1. We take

$$
G(u)=\|u\|_{D}^{2}-2 \int_{\Omega} F(x, u) d x .
$$

Under our hypotheses, Propositions 3.1-3.3 apply, and

$$
\left(G^{\prime}(u), v\right)=2(u, v)_{D}-2(f(\cdot, u), v), \quad u, v \in D .
$$

By Theorem 3.4 we see that there are positive constants $\varepsilon, \rho$ such that

$$
G(u) \geq \varepsilon, \quad\|u\|_{D}=\rho
$$

unless

$$
A u=\lambda_{0} u=f(x, u), u \in D \backslash\{0\}
$$

has a solution. This would give a nontrivial solution of (18). We may therefore assume that (30) holds. Next we note that

$G\left( \pm R \varphi_{0}\right) / R^{2}=\left\|\varphi_{0}\right\|_{D}^{2}-2 \int_{\Omega}\left\{F\left(x, \pm R \varphi_{0}\right) / R^{2} \varphi_{0}^{2}\right\} \varphi_{0}^{2} d x \rightarrow-\infty$ as $R \rightarrow \infty$

by Hypothesis (D), since $\varphi_{0} \neq 0$ a.e. Since $G(0)=0$ and (30) holds, we can now apply the usual mountain pass theorem (cf., e.g., [5, p. 22]) to conclude that there is a sequence $\left\{u_{k}\right\} \subset D$ such that

$$
G\left(u_{k}\right) \rightarrow c \geq \varepsilon, \quad G^{\prime}\left(u_{k}\right) \rightarrow 0 .
$$

Then

$$
G\left(u_{k}\right)=\rho_{k}^{2}-2 \int F\left(x, u_{k}\right) d x \rightarrow c
$$

and

$$
\left(G^{\prime}\left(u_{k}\right), u_{k}\right)=2 \rho_{k}^{2}-2\left(f\left(\cdot, u_{k}\right), u_{k}\right)=o\left(\rho_{k}\right),
$$

where $\rho_{k}=\left\|u_{k}\right\|_{D}$. Assume that $\rho_{k} \rightarrow \infty$, and let $\widetilde{u}_{k}=u_{k} / \rho_{k}$. Since $\left\|\widetilde{u}_{k}\right\|_{D}=1$, there is a renamed subsequence such that $\widetilde{u}_{k} \rightarrow \widetilde{u}$ weakly in $D$, strongly in $L_{\text {loc }}^{2}(\Omega)$ and a.e. in $\Omega$. By $(32)$,

$$
\int_{\Omega} \frac{2 F\left(x, u_{k}\right)}{u_{k}^{2}} \widetilde{u}_{k}^{2} d x \rightarrow 1
$$

Let

$$
\Omega_{1}=\{x \in \Omega: \widetilde{u}(x) \neq 0\}, \quad \Omega_{2}=\Omega \backslash \Omega_{1} .
$$

Then

$$
\frac{2 F\left(x, u_{k}\right)}{u_{k}^{2}} \widetilde{u}_{k}^{2} \rightarrow \infty, \quad x \in \Omega_{1}
$$

by Hypothesis (D). If $\Omega_{1}$ has positive measure, then

$$
\int_{\Omega} \frac{2 F\left(x, u_{k}\right)}{u_{k}^{2}} \widetilde{u}_{k}^{2} d x \geq \int_{\Omega_{1}} \frac{2 F\left(x, u_{k}\right)}{u_{k}^{2}} \widetilde{u}_{k}^{2} d x+\int_{\Omega_{2}} W(x) d x \rightarrow \infty .
$$


Thus, the measure of $\Omega_{1}$ must be 0 , i.e., we must have $\widetilde{u} \equiv 0$ a.e. Moreover,

$$
\int_{\Omega} \frac{\mu F\left(x, u_{k}\right)-u_{k} f\left(x, u_{k}\right)}{u_{k}^{2}} \widetilde{u}_{k}^{2} d x \rightarrow \frac{\mu}{2}-1 .
$$

But by Hypothesis (E),

$$
\limsup \frac{\mu F\left(x, u_{k}\right)-u_{k} f\left(x, u_{k}\right)}{u_{k}^{2}} \widetilde{u}_{k}^{2} \leq \lim \sup C \frac{u_{k}^{2}+1}{u_{k}^{2}} \widetilde{u}_{k}^{2}=0,
$$

which implies that $(\mu / 2)-1 \leq 0$, contrary to assumption. Hence, the $\rho_{k}$ are bounded. We can now follow the usual procedures to obtain a weak solution of (18) satisfying $G(u)=c \geq \varepsilon$ (cf., e.g., [5, p. 64]). Since $G(0)=0$, we see that $u \neq 0$. This completes the proof.

We postpone the proof of Theorem 2.2 until the next section.

In proving Theorem 2.3, we shall make use of:

Lemma 4.1. Under Hypothesis $\left(\mathrm{C}^{\prime}\right)$, there is an $\alpha \neq 0$ such that $G\left(\alpha \varphi_{0}\right)<$ 0 .

Proof. We can assume that

$$
\left\|\varphi_{0}\right\|_{D}=1
$$

Thus,

$$
\begin{aligned}
G\left(\alpha \varphi_{0}\right)= & \alpha^{2}-2 \int_{\Omega} F\left(x, \alpha \varphi_{0}\right) d x \\
\leq & \alpha^{2}-\widetilde{\lambda} \alpha^{2} \int_{\left|\alpha \varphi_{0}(x)\right|<\delta} \varphi_{0}(x)^{2} d x \\
& +\int_{\left|\alpha \varphi_{0}(x)\right|>\delta} V^{q}\left(\left|\alpha \varphi_{0}\right|^{q}+\left|\alpha \varphi_{0}\right|\right) \\
\leq & \alpha^{2}-\widetilde{\lambda} \alpha^{2}\left\|\varphi_{0}\right\|^{2}+C|\alpha|^{q}\left\|V \varphi_{0}\right\|_{q}^{q} \\
\leq & \alpha^{2}\left[1-\left(\widetilde{\lambda} / \lambda_{0}\right)+C^{\prime}|\alpha|^{q-2}\right] .
\end{aligned}
$$

This can be made negative by taking $\alpha$ sufficiently small.

Lemma 4.2. Under Hypothesis $\left(\mathrm{D}^{\prime}\right)$,

$$
G(u) \rightarrow \infty \text { as }\|u\|_{D} \rightarrow \infty .
$$

Proof. Suppose there is a sequence $\left\{u_{k}\right\} \subset D$ such that $\rho_{k}=\left\|u_{k}\right\| \rightarrow \infty$ and

$$
G\left(u_{k}\right) \leq K
$$

Write

$$
u_{k}=w_{k}+\alpha_{k} \varphi_{0}, \quad \tilde{u}_{k}=u_{k} / \rho_{k}, \quad \widetilde{w}_{k}=w_{k} / \rho_{k}, \quad \widetilde{\alpha}_{k}=\alpha_{k} / \rho_{k},
$$


where $w_{k} \perp \varphi_{0}$. If $\lambda_{1}>\lambda_{0}$ is the next point in the spectrum of $A$, then

$$
\lambda_{1}\|w\|^{2} \leq\|w\|_{D}^{2}, \quad w \perp \varphi_{0} .
$$

Thus

$$
\begin{aligned}
G\left(u_{k}\right) & =\left\|u_{k}\right\|_{D}^{2}-\lambda_{0}\left\|u_{k}\right\|^{2}-2 \int_{\Omega} P\left(x, u_{k}\right) d x \\
& \geq\left(1-\frac{\lambda_{0}}{\lambda_{1}}\right)\left\|w_{k}\right\|_{D}^{2}-2 \int_{\Omega} P\left(x, u_{k}\right) d x \\
& \geq\left(1-\frac{\lambda_{0}}{\lambda_{1}}\right)\left\|w_{k}\right\|_{D}^{2}-2 \int_{\Omega} W(x) d x .
\end{aligned}
$$

The only way this would not converge to $\infty$ is if $\left\|w_{k}\right\|_{D}$ is bounded. But then $\left\|\widetilde{w}_{k}\right\|_{D} \rightarrow 0$, and $\left|\widetilde{\alpha}_{k}\right| \rightarrow 1$. Since $\left\|\widetilde{u}_{k}\right\|_{D}=1$, there is a renamed subsequence such that $\widetilde{u}_{k} \rightarrow \widetilde{u}$ weakly in $D$, strongly in $L_{\text {loc }}^{2}(\Omega)$ and a.e. in $\Omega$. Since $\widetilde{w}=0$ and $|\widetilde{\alpha}|=1$, we have $\widetilde{u}(x)=\widetilde{\alpha} \varphi_{0}(x) \neq 0$ a.e. Hence, $\left|u_{k}(x)\right|=\rho_{k}\left|\widetilde{u}_{k}(x)\right| \rightarrow \infty$ a.e. Consequently,

$$
\int_{\Omega} P\left(x, u_{k}\right) d x \rightarrow-\infty
$$

showing that $G\left(u_{k}\right) \rightarrow \infty$. This completes the proof.

We can now give:

Proof of Theorem 2.3. Let

$$
m=\inf _{D} G .
$$

Then there is a sequence $\left\{u_{k}\right\} \subset D$ such that $G\left(u_{k}\right) \rightarrow m$. In view of Lemma 4.2 , we must have $\left\|u_{k}\right\|_{D} \leq C$. Thus, there is a renamed subsequence such that $u_{k} \rightarrow u$ weakly in $D$, strongly in $L_{\text {loc }}^{2}(\Omega)$ and a.e. in $\Omega$. Now,

$$
\begin{aligned}
G(u)= & \|u\|_{D}^{2}-2 \int_{\Omega} F(x, u) d x \\
= & \left\|u_{k}\right\|_{D}^{2}-2\left(\left[u_{k}-u\right], u\right)_{D}-\left\|u_{k}-u\right\|_{D}^{2} \\
& -2 \int_{\Omega} F\left(x, u_{k}\right) d x+2 \int_{\Omega}\left[F\left(x, u_{k}\right)-F(x, u)\right] d x \\
\leq & G\left(u_{k}\right)-2\left(\left[u_{k}-u\right], u\right)_{D}+2 \int_{\Omega}\left[F\left(x, u_{k}\right)-F(x, u)\right] d x .
\end{aligned}
$$

From our hypotheses, it follows that

$$
\int_{\Omega} F\left(x, u_{k}\right) d x \rightarrow \int_{\Omega} F(x, u) d x
$$

(cf., e.g., [5, p. 64]). We therefore have in the limit $G(u) \leq m$, from which we conclude that $G(u)=m$ and $G^{\prime}(u)=0$. Hence, $u$ is a weak solution of (10). We see from Lemma 4.1 that $m<0$. Since $G(0)=0$, we see that $u \neq 0$. This completes the proof. 


\section{The eigenvalue problem.}

In this section we shall give the proofs of Theorems 2.4, 2.5 and 2.2. They will be based on the following result given in [7]. Let $E$ be a reflexive Banach space with norm $\|\cdot\|$, and let $A, B$ be two closed subsets of $E$. Suppose that $G \in C^{1}(E, \mathbb{R})$ is of the form: $G(u):=I(u)-J(u), u \in E$, where $I, J \in C^{1}(E, \mathbb{R})$ map bounded sets to bounded sets. Define

$$
G_{\lambda}(u)=\lambda I(u)-J(u), \quad \lambda \in \Lambda,
$$

where $\Lambda$ is an open interval contained in $(0,+\infty)$. Assume one of the following alternatives holds:

$\left(\mathrm{H}_{1}\right) I(u) \geq 0$ for all $u \in E$ and either $I(u) \rightarrow \infty$ or $|J(u)| \rightarrow \infty$ as $\|u\| \rightarrow \infty$.

$\left(\mathrm{H}_{2}\right) I(u) \leq 0$ for all $u \in E$ and either $I(u) \rightarrow-\infty$ or $|J(u)| \rightarrow \infty$ as $\|u\| \rightarrow \infty$.

Furthermore, we suppose that:

$\left(\mathrm{H}_{3}\right) a_{0}(\lambda):=\sup _{A} G_{\lambda} \leq b_{0}(\lambda):=\inf _{B} G_{\lambda}$, for any $\lambda \in \Lambda$.

We let $\Phi$ be the set of mappings $\Gamma(t) \in C(E \times[0,1], E)$ with the following properties:

a) for each $t \in[0,1), \Gamma(t)$ is a homeomorphism of $E$ onto itself and $\Gamma(t)^{-1}$ is continuous on $E \times[0,1)$;

b) $\Gamma(0)=I$;

c) for each $\Gamma(t) \in \Phi$ there is a $u_{0} \in E$ such that $\Gamma(1) u=u_{0}$ for all $u \in E$ and $\Gamma(t) u \rightarrow u_{0}$ as $t \rightarrow 1$ uniformly on bounded subsets of $E$.

A subset $A$ of $E$ links a subset $B$ of $E$ if $A \cap B=\phi$ and, for each $\Gamma(t) \in \Phi$, there is a $t \in(0,1]$ such that $\Gamma(t) A \cap B \neq \phi$.

We have:

Theorem 5.1. Assume that $\left(\mathrm{H}_{1}\right)$ (or $\left.\left(\mathrm{H}_{2}\right)\right)$ and $\left(\mathrm{H}_{3}\right)$ hold.

(1) If $A$ links $B$ and $A$ is bounded, then for almost all $\lambda \in \Lambda$ there exists $u_{k}(\lambda) \in E$ such that $\sup _{k}\left\|u_{k}(\lambda)\right\|<\infty, G_{\lambda}^{\prime}\left(u_{k}(\lambda)\right) \rightarrow 0$ and

$$
G_{\lambda}\left(u_{k}(\lambda)\right) \rightarrow a(\lambda):=\inf _{\Gamma \in \Phi} \sup _{s \in[0,1], u \in A} G_{\lambda}(\Gamma(s, u)), \quad k \rightarrow \infty .
$$

Furthermore, if $a(\lambda)=b_{0}(\lambda)$, then $\operatorname{dist}\left(u_{k}(\lambda), B\right) \rightarrow 0, \quad k \rightarrow \infty$.

(2) If $B$ links $A$ and $B$ is bounded, then for almost all $\lambda \in \Lambda$ there exists $v_{k}(\lambda) \in E$ such that $\sup _{k}\left\|v_{k}(\lambda)\right\|<\infty, G_{\lambda}^{\prime}\left(v_{k}(\lambda)\right) \rightarrow 0$ and

$$
G_{\lambda}\left(v_{k}(\lambda)\right) \rightarrow b(\lambda):=\sup _{\Gamma \in \Phi} \inf _{s \in[0,1], v \in B} G_{\lambda}(\Gamma(s, v)), \quad k \rightarrow \infty .
$$

Furthermore, if $a_{0}(\lambda)=b(\lambda)$, then $\operatorname{dist}\left(v_{k}(\lambda), A\right) \rightarrow 0, \quad k \rightarrow \infty$.

We shall also need the following extension of Theorem 3.4: 
Theorem 5.2. Let $\lambda$ be a parameter satisfying $1<\lambda \leq K<\infty$. Under Hypotheses (A)-(D), for each $\rho>0$ sufficiently small (not depending on $\lambda$ ), we have

$$
G_{\lambda}(u):=\lambda\|u\|_{D}^{2}-2 \int_{\Omega} F(x, u) d x \geq(\lambda-1) \rho^{2}, \quad\|u\|_{D}=\rho .
$$

If we replace Hypothesis $(\mathrm{C})$ with Hypothesis $\left(\mathrm{C}^{\prime \prime}\right)$, assuming $1<\widetilde{\lambda} / \lambda_{0}<$ $\lambda \leq K<\infty$, then we have

$$
G_{\lambda}(u) \geq\left(\lambda-\frac{\tilde{\lambda}}{\lambda_{0}}\right) \rho^{2}, \quad\|u\|_{D}=\rho .
$$

Proof. Let $\lambda_{1}>\lambda_{0}$ be the next point in the spectrum of $A$, and let $N_{0}$ denote the eigenspace of $\lambda_{0}$. We take $M=N_{0}^{\perp} \cap D$. By Hypothesis (B), there is a $\rho>0$ such that

$$
\|y\|_{D} \leq \rho \Rightarrow|y(x)| \leq \delta / 2, \quad y \in N_{0} .
$$

Now suppose $u \in D$ satisfies

$$
\|u\|_{D} \leq \rho \text { and }|u(x)| \geq \delta
$$

for some $x \in \Omega$. We write

$$
u=w+y, w \in M, y \in N_{0} .
$$

Then for those $x \in \Omega$ satisfying (38) we have

$$
\delta \leq|u(x)| \leq|w(x)|+|y(x)| \leq|w(x)|+(\delta / 2) .
$$

Hence

$$
|y(x)| \leq \delta / 2 \leq|w(x)|
$$

and consequently,

$$
|u(x)| \leq 2|w(x)|
$$

for all such $x$. Now we have by (12) and (14)

$$
\begin{aligned}
G_{\lambda}(u) & \geq \lambda\|u\|_{D}^{2}-\lambda_{0} \int_{|u|<\delta} u^{2} d x-C \int_{|u|>\delta}\left(|V u|^{q}+V^{q}|u|\right) d x \\
& \geq \lambda\|u\|_{D}^{2}-\lambda_{0}\|u\|^{2}-C^{\prime} \int_{|u|>\delta}|V u|^{q} d x \\
& \geq(\lambda-1)\|y\|_{D}^{2}+\lambda\|w\|_{D}^{2}-\lambda_{0}\|w\|^{2}-C^{\prime \prime} \int_{2|w|>\delta}|V w|^{q} d x
\end{aligned}
$$

in view of the fact that $\|y\|_{D}^{2}=\lambda_{0}\|y\|^{2}$ and (41) holds. Thus, by (14),

$$
G_{\lambda}(u) \geq(\lambda-1)\|y\|_{D}^{2}+\left(\lambda-\frac{\lambda_{0}}{\lambda_{1}}-C^{\prime \prime \prime}\|w\|_{D}^{q-2}\right)\|w\|_{D}^{2}, \quad\|u\|_{D} \leq \rho .
$$


We take $\rho>0$ to satisfy

$$
1-\frac{\lambda_{0}}{\lambda_{1}}>C^{\prime \prime \prime} \rho^{q-2}
$$

This gives

$$
\begin{aligned}
G_{\lambda}(u) & \geq(\lambda-1) \rho^{2}+\left(\lambda-\frac{\lambda_{0}}{\lambda_{1}}-C^{\prime \prime \prime} \rho^{q-2}-\lambda+1\right)\|w\|_{D}^{2} \\
& \geq(\lambda-1) \rho^{2}, \quad\|u\|_{D}=\rho .
\end{aligned}
$$

Hence, (36) holds.

To prove (37) under Hypothesis $\left(\mathrm{C}^{\prime \prime}\right)$, let $\eta=\widetilde{\lambda} / \lambda_{0}$ and $\Lambda=(\eta, K)$. Under Hypothesis $\left(\mathrm{C}^{\prime \prime}\right)$ we have in place of $(42)$

$$
G_{\lambda}(u) \geq(\lambda-\eta)\|y\|_{D}^{2}+\left(\lambda-\frac{\widetilde{\lambda}}{\lambda_{1}}-C^{\prime \prime \prime}\|w\|_{D}^{q-2}\right)\|w\|_{D}^{2}, \quad\|u\|_{D} \leq \rho .
$$

We take $\rho>0$ to satisfy

$$
\eta-\frac{\widetilde{\lambda}}{\lambda_{1}}>C^{\prime \prime \prime \prime} \rho^{q-2}
$$

Consequently,

$$
\begin{aligned}
G_{\lambda}(u) & \geq(\lambda-\eta) \rho^{2}+\left(\lambda-\frac{\tilde{\lambda}}{\lambda_{1}}-C^{\prime \prime \prime} \rho^{q-2}-\lambda+\eta\right)\|w\|_{D}^{2} \\
& \geq(\lambda-\eta) \rho^{2}, \quad\|u\|_{D}=\rho .
\end{aligned}
$$

This gives (37), and the proof is complete.

We now turn to the proofs of Theorems 2.4 and 2.5. We prove the latter first. We shall prove Theorem 2.5 by applying Theorems 5.1 and 5.2.

Proof. We take $E=D, \Lambda=(\eta, K)$, where $\eta=\widetilde{\lambda} / \lambda_{0}, K>1$ is a finite number, and

$$
I(u)=\|u\|_{D}^{2}, \quad J(u)=2 \int_{\Omega} F(x, u) d x .
$$

For the purpose of this application, it is sufficient to know that the sets

$$
A_{ \pm}=\left[0, \pm R \varphi_{0}\right], \quad B=\left\{x \in D:\|x\|_{D}=\rho\right\}
$$

link each other if $R>\rho$ (cf., e.g., [5]). In our case Hypothesis $\left(\mathrm{H}_{1}\right)$ is satisfied. We now check that $\left(\mathrm{H}_{3}\right)$ holds. We observe that

$$
G_{\lambda}^{\prime}(u)=0
$$

is equivalent to $(23)$ with $\beta=1 / \lambda$. Now, at least one of the expressions

$$
J\left( \pm R \varphi_{0}\right) / R^{2}=2 \int_{\Omega} F\left(x, \pm R \varphi_{0}\right) d x / R^{2} \rightarrow \infty \text { as } R \rightarrow \infty
$$


by Hypothesis $\left(\mathrm{D}^{\prime \prime}\right)$. Hence, for $R$ sufficiently large, one of the inequalities

$$
G_{\lambda}\left( \pm R \varphi_{0}\right) / R^{2} \leq K\left\|\varphi_{0}\right\|_{D}^{2}-2 \int_{\Omega} F\left(x, \pm R \varphi_{0}\right) / R^{2} \leq 0
$$

holds. Thus,

$$
a_{0}(\lambda) \leq 0, \quad \lambda \in \Lambda .
$$

Moreover, it follows from Theorem 5.2 that (37) holds. Hence,

$$
b_{0}(\lambda) \geq(\lambda-\eta) \rho^{2}, \quad \lambda \in \Lambda .
$$

This shows that Hypothesis $\left(\mathrm{H}_{3}\right)$ holds. We can now apply Theorem 5.1 to conclude that for almost all $\lambda \in \Lambda$, there exists $u_{k}(\lambda) \in D$ such that $\sup _{k}\left\|u_{k}(\lambda)\right\|<\infty, G_{\lambda}^{\prime}\left(u_{k}(\lambda)\right) \rightarrow 0$ and

$$
G_{\lambda}\left(u_{k}(\lambda)\right) \rightarrow a(\lambda) \geq b_{0}(\lambda) .
$$

Once it is known that the sequence $\left\{u_{k}\right\}$ is bounded, we can apply the usual theory to conclude that there is a solution of

$$
G_{\lambda}^{\prime}(u)=0, \quad G_{\lambda}(u)=a(\lambda)
$$

(cf., e.g., [5, p. 64]). Moreover, from the definition, we see that $a(\lambda) \geq$ $(\lambda-\eta) \rho^{2}$. Hence, the equation $G_{\lambda}^{\prime}(u)=0$ has a nontrivial solution for almost every $\lambda \in \Lambda$. This is equivalent to (23) having a nontrivial solution for almost every $\beta \in\left(K^{-1}, \eta^{-1}\right)$. Since $K$ was arbitrary, the result follows.

To prove Theorem 2.4, it suffices to take $\widetilde{\lambda}=\lambda_{0}$ and show that Hypothesis (D) implies Hypothesis $\left(\mathrm{D}^{\prime \prime}\right)$. To see this, we note that

$$
\int_{\Omega} F\left(x, \pm R \varphi_{0}\right) d x / R^{2}=\int_{\Omega} \frac{F\left(x, \pm R \varphi_{0}\right)}{R^{2} \varphi_{0}^{2}} \varphi_{0}^{2} d x \rightarrow \infty
$$

by Hypothesis (D) and the fact that $\varphi_{0}(x) \neq 0$ a.e.

To prove Corollary 2.6, we let $\varepsilon$ be any positive number. By Hypothesis $\left(\mathrm{C}^{\prime \prime \prime}\right)$, there is a $\delta>0$ such that

$$
F(x, t) / t^{2} \leq \varepsilon, \quad|t| \leq \delta, \quad x \in \Omega .
$$

By Theorem 2.5, Equation (23) has a nontrivial solution for a.e. $\beta \in\left(0, \lambda_{0} / \varepsilon\right)$. Since $\varepsilon$ was arbitrary, the result follows.

We now give the proof of Theorem 2.2.

Proof. By Theorem 2.4, for each arbitrary $K>1$, and a.e. $\lambda \in(1, K)$, there exists $u_{\lambda}$ such that $G_{\lambda}^{\prime}\left(u_{\lambda}\right)=0, \quad G_{\lambda}\left(u_{\lambda}\right)=a(\lambda) \geq(\lambda-1) \rho^{2}$. Choose $\lambda_{n} \rightarrow 1, \lambda_{n}>1$. Then there exists $u_{n}$ such that

$$
G_{\lambda_{n}}^{\prime}\left(u_{n}\right)=0, \quad G_{\lambda_{n}}\left(u_{n}\right)=a\left(\lambda_{n}\right) \geq a(1) \geq b_{0}(1) .
$$


By Theorem 3.4, we may assume $b_{0}(1) \geq \varepsilon>0$. Therefore,

$$
\int_{\Omega} \frac{2 F\left(x, u_{n}\right)}{\left\|u_{n}\right\|_{D}^{2}} d x \leq c .
$$

Now we prove that $\left\{u_{n}\right\}$ is bounded. If $\left\|u_{n}\right\|_{D} \rightarrow \infty$, let $w_{n}=u_{n} /\left\|u_{n}\right\|_{D}$, then $w_{n} \rightarrow w$ weakly in $D$, strongly in $L_{\text {loc }}^{2}(\Omega)$ and a.e. in $\Omega$.

Case 1: $w \neq 0$ in $D$. We get a contradiction as follows:

$$
\begin{aligned}
c & \geq \int_{\Omega} \frac{2 F\left(x, u_{n}\right)}{\left\|u_{n}\right\|_{D}^{2}} d x=\int_{\Omega} \frac{2 F\left(x, u_{n}\right)}{u_{n}^{2}}\left|w_{n}\right|^{2} d x \\
& \geq \int_{w \neq 0} \frac{2 F\left(x, u_{n}\right)}{u_{n}^{2}}\left|w_{n}\right|^{2} d x-\int_{w=0} W_{1}(x) d x \rightarrow \infty .
\end{aligned}
$$

Case 2: $w=0$ in $D$. We define $t_{n} \in[0,1]$ by

$$
G_{\lambda_{n}}\left(t_{n} u_{n}\right)=\max _{t \in[0,1]} G_{\lambda_{n}}\left(t u_{n}\right) .
$$

For any $c>0$ and $\bar{w}_{n}=c w_{n}$, we have

$$
\int_{\Omega} F\left(x, \bar{w}_{n}\right) d x \rightarrow 0
$$

(cf., e.g., [5, p. 64]). Thus,

$$
G_{\lambda_{n}}\left(t_{n} u_{n}\right) \geq G_{\lambda_{n}}\left(c w_{n}\right)=c^{2} \lambda_{n}-2 \int_{\Omega} F\left(x, \bar{w}_{n}\right) d x \geq c^{2} / 2
$$

for $n$ large enough. That is, $\lim _{n \rightarrow \infty} G_{\lambda_{n}}\left(t_{n} u_{n}\right)=\infty$ and $\left(G_{\lambda_{n}}^{\prime}\left(t_{n} u_{n}\right), u_{n}\right)=0$. Therefore,

$$
\begin{aligned}
G_{\lambda_{n}}\left(t_{n} u_{n}\right) & =\int_{\Omega}\left(f\left(x, t_{n} u_{n}\right) t_{n} u_{n}-2 F\left(x, t_{n} u_{n}\right)\right) d x \\
& =\int_{\Omega} H\left(x, t_{n} u_{n}\right) d x \rightarrow \infty .
\end{aligned}
$$

By Hypothesis $\left(\mathrm{E}^{\prime}\right)$,

$$
G_{\lambda_{n}}\left(u_{n}\right)=\int_{\Omega} H\left(x, u_{n}\right) d x \geq \int_{\Omega} H\left(x, t_{n} u_{n}\right) d x \rightarrow \infty .
$$

But

$$
\begin{aligned}
G_{\lambda_{n}}\left(u_{n}\right)=a\left(\lambda_{n}\right) & \leq \sup _{s \in[0,1], u \in A} G_{\lambda_{n}}((1-s) u) \\
& \leq \sup _{s \in[0,1], u \in A} G_{K}((1-s) u) \\
& <c
\end{aligned}
$$


a contradiction. Thus, $\left\|u_{n}\right\|_{D} \leq C$. It now follows that

$$
G^{\prime}\left(u_{n}\right) \rightarrow 0, \quad G\left(u_{n}\right) \rightarrow a(1) \geq b_{0}(1) .
$$

We can now apply Theorem 3.4.1 in [5, p. 64] to obtain the desired solution.

\section{References}

[1] A. Ambrosetti and P.H. Rabinowitz, Dual variational methods in critical point theory and applications, J. Funct. Anal., 14 (1973), 349-381, MR 0370183, Zbl 0273.49063.

[2] D.G. Costa and C.A. Magalhães, Variational elliptic problems which are nonquadratic at infinity, Nonlinear Anal., 23 (1994), 1401-1412, MR 1306679, Zbl 0820.35059.

[3] L. Jeanjean, On the existence of bounded Palais-Smale sequences and applications to a Landesman-Lazer type problem set on $\mathbb{R}^{N}$, Proc. Roy. Soc. Edinburgh A, 129 (1999), 787-809, MR 1718530, Zbl 0935.35044.

[4] M. Schechter, Superlinear elliptic boundary value problems, Manuscripta Math., 86 (1995) 253-265, MR 1323791, Zbl 0839.35048.

[5] — Linking Methods in Critical Point Theory, Birkhäuser, Boston, 1999, MR 1729208, Zbl 0915.35001.

[6] _ Spectra of Partial Differential Operators, North-Holland, Amsterdam, 1986, MR 0869254, Zbl 0607.35005.

[7] M. Schechter and W. Zou, Double linking theorem and multiple solutions, J. Funct. Anal., 205 (2003), 37-61.

[8] M. Struwe, The existence of surfaces of constant mean curvature with free boundaries, Acta Math., 160 (1988) 19-64, MR 0926524, Zbl 0646.53005.

[9] _ Variational Methods, Ergebnisse Math. Grenz., 34, Springer, Berlin, 1996, MR 1411681, Zbl 0864.49001.

[10] M. Willem, Minimax Theorems, Birkhäuser, Boston, 1996, MR 1400007, Zbl 0856.49001.

[11] M. Willem and W. Zou, On a semilinear Dirichlet problem and a nonlinear Schrödinger equation with periodic potential, Indiana Univ. Math. J., 52 (2003), 109132 .

Received November 10, 2002. The first author was supported in part by an NSF grant and the second author was supported by NSFC grant (GP10001019). Zou thanks the Mathematics Department of the University of California at Irvine for an appointment to the department for the years 2001-2004.

Department of Mathematics

UNIVERSITY OF CALIFORNIA

IRvine, CA 92697-3875

E-mail address: mschecht@math.uci.edu

Department of Mathematical Sciences

TSInghua University

BEIJING 100084

P.R. China

E-mail address: wzou@math.tsinghua.edu.cn 